Pure and Applied Mathematics Quarterly

Volume 8, Number 1

(Special Issue: In honor of

F. Thomas Farrell and Lowell E. Jones, Part 1 of 2)

79-105, 2012

\title{
Remarks Concerning Lubotzky's Filtration
}

\author{
F. R. Cohen*, Marston Conder, J. Lopez and Stratos Prassidis ${ }^{\dagger}$
}

Abstract: A discrete group which admits a faithful, finite dimensional, linear representation over a field $\mathbb{F}$ of characteristic zero is called linear. This note combines the natural structure of semi-direct products with work of A. Lubotzky [13] on the existence of linear representations to develop a technique to give sufficient conditions to show that a semi-direct product is linear.

Let $G$ denote a discrete group which is a semi-direct product given by a split extension

$$
1 \rightarrow \pi \rightarrow G \rightarrow \Gamma \rightarrow 1
$$

This note defines an additional type of structure for this semi-direct product called a stable extension below. The main results are as follows:

(1) If $\pi$ and $\Gamma$ are linear, and the extension is stable, then $G$ is also linear. Restrictions concerning this extension are necessary to guarantee that $G$ is linear as seen from properties of the Formanek-Procesi "poison group" [7].

(2) If the action of $\Gamma$ on $\pi$ has a "Galois-like" property that it factors through the automorphisms of certain natural "towers of groups over $\pi$ " ( to be defined below ), then the associated extension is stable and thus $G$ is linear.

(3) The condition of a stable extension also implies that $G$ admits filtration quotients which themselves give a natural structure of Lie algebra and

Received September 29, 2007.

*Partially supported by the NSF

${ }^{\dagger}$ Partially supported by Canisius College Summer Grant 
which also imply earlier results of Kohno, and Falk-Randell $[10,6]$ on the Lie algebra attached to the descending central series associated to the fundamental groups of complex hyperplane complements.

The methods here suggest that a possible technique for obtaining new linearity results may be to analyze automorphisms of towers of groups.

Keywords: Semi-Direct Products, Faithful Representations, Filtrations, Lie Algebras.

\section{INTRODUCTION}

A. Lubotzky [13] or [5], pages 172-175, gave a purely group theoretic criterion which is equivalent to the existence a faithful finite dimensional representation over a field $\mathbb{F}$ of characteristic zero for a discrete group $G$ (where the image is not necessarily discrete). A group $G$ with this property is called linear.

The purpose of this paper is to give an extension of Lubotzky's criterion which can sometimes be applied to show that a semi-direct product of linear groups is again linear. The main subject of this article is a split extension of groups given by

$$
1 \longrightarrow \pi \stackrel{i}{\longrightarrow} G \stackrel{p}{\longrightarrow} \Gamma \longrightarrow 1
$$

for which it is assumed that both $\pi$ and $\Gamma$ are linear. The main purpose of this article is to define the notion of a stable extension as given in Definition 2.5 which implies that $G$ is linear.

The approach weaves together semi-direct products regarded as pull-backs of a universal semi-direct product known as the holomorph together with certain choices of filtrations of both $\pi$ and $\Gamma$. Roughly speaking, one of the main results here is that representations of $\Gamma$ in the automorphism group of $\pi$ which factor through the automorphism group of the filtration of $\pi$ as given in Definition 3.1 suffices to show that $G$ is linear via Lubotzky's criteria [13].

Notice that it may be the case that both $\pi$ and $\Gamma$ admit faithful finite dimensional representations, but that $G$ does not. A basic example due to Formanek and Procesi [7] is a split extension

$$
1 \longrightarrow F_{3} \stackrel{i}{\longrightarrow} H \stackrel{p}{\longrightarrow} F_{2} \longrightarrow 1
$$


where $H=G, F_{n}$ is a free group on $n$ letters, and the group $H$ admits the following presentation:

$$
H=\left\langle a_{1}, a_{2}, a_{3}, \phi_{1}, \phi_{2} \mid \phi_{i} a_{j} \phi_{i}^{-1}=a_{j}, \phi_{i} a_{3} \phi_{i}^{-1}=a_{3} a_{i}, i, j=1,2\right\rangle .
$$

This example, the Formanek-Procesi "poison group", is a subgroup of $A u t\left(F_{3}\right)$, the automorphism group of $F_{3}$ and has the property that the action of $F_{2}$ on the first homology group of $F_{3}$ is non-trivial.

Contrasting examples with $\pi$ given by $F_{n}$ which do in fact admit faithful finite dimensional representations from the methods given here are explained next. A subgroup of $\operatorname{Aut}\left(F_{n}\right)$ known as McCool's group $M(n)$ is generated by automorphisms given by conjugating a fixed basis element by another fixed basis element [14]. Furthermore, the kernel of the natural map $\operatorname{Aut}\left(F_{n}\right) \rightarrow G L(n, \mathbb{Z}), I A_{n}$, contains $M(n)$.

Consider a split extension

$$
1 \longrightarrow F_{n} \stackrel{i}{\longrightarrow} G \stackrel{p}{\longrightarrow} \Gamma \longrightarrow 1
$$

where $\Gamma$ admits a faithful finite dimensional representation and the action of $\Gamma$ on $F_{n}$ factors through $M(n)$. It is shown below that $G$ is sometimes linear. Thus it is natural to ask the following question which is also raised in [2] with some additional evidence here.

Conjecture 1.1. Consider a split exact sequence of groups

$$
1 \longrightarrow F_{n} \longrightarrow G \longrightarrow \Gamma \longrightarrow 1
$$

with $F_{n}$ a free group on $n$ letters and $\Gamma$ a group that admits a finite dimensional faithful linear representation. If the conjugation action of $\Gamma$ on $F_{n}$ is trivial on homology, $H_{1}\left(F_{n} ; \mathbb{Z}\right)$, and thus factors through $I A_{n}$, then $G$ is linear.

A weaker conjecture is to replace $I A_{n}$ by McCool's group $M(n)$ : that is, if the conjugation action of $\Gamma$ on $F_{n}$ factors through $M(n)$, then $G$ is linear.

Notice that both conjectures are consistent with work of Bigelow [1] and Krammer [11] who proved that Artin's braid groups are linear. The veracity of either conjecture implies that the pure braid groups, thus the full braid groups are linear.

Remark 1.2. Observe that $M(2)=I A_{2}$. Thus in case $n=2$, this conjecture follows directly from the observations in Corollary 8.3 below. In case $\Gamma$ is a subgroup of $G L(n, \mathbb{F})$, it follows from the computations below that $G$ is a subgroup of $G L(n+4, \mathbb{F})$ with details left as an exercise. 
The authors would like to congratulate Tom Farrell and Lowell Jones on this happy occasion of their 60-th birthday. The authors would also like to thank the organizers for this stimulating and interesting opportunity to participate in an excellent conference.

\section{Definitions and Statement of Results}

Recall the following definition from [5] (page 171) and [13].

Definition 2.1. A filtration of the group $\pi$ is a descending chain of normal subgroups

$$
\cdots \subseteq L_{j}(\pi) \subseteq \cdots \subseteq L_{1}(\pi) \subseteq L_{0}(\pi)=\pi
$$

for $j \geq 0$ such that $\bigcap_{j \geq 1} L_{j}(\pi)=\{1\}$.

Definition 2.2. A $p$-congruence system for the group $\pi$ is a filtration of $\pi$

$$
\cdots \subseteq L_{j}(\pi) \subseteq \cdots \subseteq L_{1}(\pi) \subseteq L_{0}(\pi)=\pi
$$

for $j \geq 0$ such that

(1) $\pi / L_{1}(\pi)$ is finite, and

(2) $L_{1}(\pi) / L_{1+j}(\pi)$ is a finite $p$-group for all $j \geq 0$.

Definition 2.3. A bounded p-congruence system for the group $\pi$ is a $p$-congruence system for the group $\pi$ given by

$$
\cdots \subseteq L_{j}(\pi) \subseteq \cdots \subseteq L_{1}(\pi) \subseteq L_{0}(\pi)=\pi
$$

such that $d\left(L_{i}(\pi) / L_{j}(\pi)\right) \leq e$ for all $0 \leq i<j$ (where the number $d(G)$ denotes the minimal number of generators of the group $G$ ([5], page xvii)). A bounded $p$-congruence system is also called a Lubotzky filtration below.

The following is a restatement here of a result due to A. Lubotzky [13].

Theorem 2.4. A group $G$ admits a bounded p-congruence system for some prime $p$ if and only if $G$ admits a faithful finite dimensional representation for some field of characteristic zero.

Let $\operatorname{Aut}(\pi)$ denote the automorphism group of $\pi$. Consider a discrete group $\pi$ together with the universal semi-direct product $\operatorname{Hol}(\pi)$ "the natural" split extension of $\operatorname{Aut}(\pi)$ by $\pi$,

$$
1 \rightarrow \pi \rightarrow \operatorname{Hol}(\pi) \rightarrow \operatorname{Aut}(\pi) \rightarrow 1
$$


The group $\operatorname{Hol}(\pi)$, as a set, is the product $\operatorname{Aut}(\pi) \times \pi$ with the product structure defined by the formula

$$
(f, x) \cdot(g, y)=\left(f \cdot g, g^{-1}(x) \cdot y\right)
$$

for $f, g$ in $\operatorname{Aut}(\pi)$, and $x, y$ in $\pi$.

The next four formulas follow from the definition but are listed here for convenience of the reader in the proofs below.

(1) $(f, x)^{-1}=\left(f^{-1}, f\left(x^{-1}\right)\right)$,

(2) $(f, 1)^{-1} \cdot(1, y) \cdot(f, 1)=\left(1, f^{-1}(y)\right)$

(3) $(f, x) \cdot(g, y) \cdot(f, x)^{-1}=\left(f \cdot g \cdot f^{-1}, f\left(g^{-1}(x) \cdot y\right) \cdot f\left(x^{-1}\right)\right)$, and

(4) $[(f, x),(g, y)]=\left(f \cdot g \cdot f^{-1} \cdot g^{-1}, g\left\{f\left(g^{-1}(x) \cdot y\right) \cdot f\left(x^{-1}\right)\right\} \cdot g\left(y^{-1}\right)\right)$.

Consider a homomorphism

$$
\phi: \Gamma \rightarrow \operatorname{Aut}(\pi)
$$

called the classifying map for the extension. Pull back the extension determined by $\operatorname{Hol}(\pi)$ to obtain the extension $G$ together with a morphism of extensions (as developed in more detail in [16]):

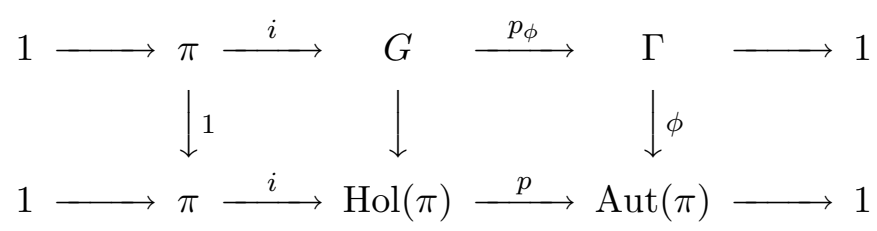

Furthermore, every split extension of $\Gamma$ with kernel $\pi$ is given by such a pull-back for some choice of homomorphism

$$
\phi: \Gamma \rightarrow \operatorname{Aut}(\pi) .
$$

Thus if $(f, x),(g, y) \in \Gamma \times \pi$ then $(f, x) \cdot(g, y)=\left(f \cdot g, \phi\left(g^{-1}\right)(x) \cdot y\right)$. A notational convention used throughout this article is that $g^{-1}(x)$ denotes $\phi\left(g^{-1}\right)(x)$.

The results here intertwine filtrations for the groups $\pi$ and $\Gamma$ in the extension

$$
1 \longrightarrow \pi \stackrel{i}{\longrightarrow} G \stackrel{p_{\phi}}{\longrightarrow} \Gamma \longrightarrow 1
$$

by focusing on the classifying map for the extension given by $\phi: \Gamma \rightarrow \operatorname{Aut}(\pi)$ rather than considering the extension itself. Thus, the main focus here are conditions concerning the homomorphism $\phi: \Gamma \rightarrow \operatorname{Aut}(\pi)$ which imply that $G$ is linear. 
Definition 2.5. Assume that

$$
1 \longrightarrow \pi \stackrel{i}{\longrightarrow} G \stackrel{p_{\phi}}{\longrightarrow} \Gamma \longrightarrow 1
$$

is a split extension classified by the map

$$
\phi: \Gamma \rightarrow \operatorname{Aut}(\pi)
$$

together with filtrations

(1) for the group $\pi$

$$
\cdots \subseteq L_{j}(\pi) \subseteq \cdots \subseteq L_{1}(\pi) \subseteq L_{0}(\pi)=\pi
$$

for $j \geq 0$ and

(2) for $\Gamma$

$$
\cdots \subseteq F_{j}(\Gamma) \subseteq \cdots \subseteq F_{1}(\Gamma) \subseteq F_{0}(\Gamma)=\Gamma
$$

for $j \geq 0$.

The extension ( together with the two filtrations) is said to be stable if and only if for every $(g, y)$ in $F_{r+s}(\Gamma) \times L_{r+s}(\pi)$ and for every $(f, x)$ in $F_{r}(\Gamma) \times L_{r}(\pi)$ the following properties are satisfied for $r, s \geq 0$ :

(1) $f(y) \in L_{r+s}(\pi)$ and

(2) $g(x)=\delta_{x} \cdot x$ for $\delta_{x} \in L_{r+s}(\pi)$.

Remark 2.6. These two conditions both of which must be satisfied in what is given below fit naturally with extensions. They arise by considering the natural "twisting" for the holomorph as well as for certain fibre bundles.

The definition of a stable extension is basically recording the feature that certain extensions "look like products" modulo certain higher filtrations. One result is as follows.

Theorem 2.7. Assume that the split extension

$$
1 \longrightarrow \pi \stackrel{i}{\longrightarrow} G \stackrel{p_{\phi}}{\longrightarrow} \Gamma \longrightarrow 1
$$

is classified by the map $\phi: \Gamma \rightarrow$ Aut $(\pi)$ which satisfies the conditions that

(1) $\Gamma$ and $\pi$ admit bounded $p$-congruence systems for some prime $p$ as given in Definition 2.3, and

(2) the p-congruence systems for the groups $\pi$ and $\Gamma$ in part (1) are stable in the sense of Definition 2.5.

Then $G$ is linear. 
Examples of Theorem 2.7 are given in sections 7 and 8 . These examples arise by forming the split extension

$$
1 \rightarrow F_{n} \rightarrow G \rightarrow \Gamma \rightarrow 1
$$

where

(1) $\Gamma$ is a subgroup of $G L(2, \mathbb{Z})$ ( and thus $\Gamma$ has a normal finite index subgroup which is free ),

(2) $F_{n}$ is isomorphic to a principal congruence subgroup of level $p^{r}$ in $P S L(2, \mathbb{Z})$, and

(3) $\Gamma$ acts by conjugation on $F_{n}$.

That these examples are linear follows from standard elementary methods as well as the methods here. One related special case is as follows.

Example 2.8. Consider the extension

$$
1 \longrightarrow F\left[a_{1}, a_{2}, \cdots, a_{n}, b\right] \longrightarrow G_{n} \longrightarrow F[x, y] \longrightarrow 1
$$

for which the action of $F[x, y]$ is given as follows.

(1) (a) $x\left(a_{q}\right)=a_{q+1}$ if $1 \leq q<n$ with $x\left(a_{n}\right)=b \cdot a_{1} \cdot b^{-1}$ and

(b) $x(b)=b$.

(2) The action of $y$ is given by

(a) $y\left(a_{q}\right)=a_{1} \cdot a_{q} \cdot a_{1}^{-1}$ and

(b) $y(b)=a_{1} \cdot b \cdot a_{1}^{-1}$.

Then $G_{n}$ is linear. As shown in Section 8 , these examples can be done easily by using elementary, "bare-hands" methods.

In the case of a split extension

$$
1 \longrightarrow \pi \stackrel{i}{\longrightarrow} G \stackrel{p_{\phi}}{\longrightarrow} \Gamma \longrightarrow 1
$$

which is stable ( Definition 2.5), the group $G$ inherits a natural filtration which is defined next with properties developed in section 4 .

Definition 2.9. A filtration of the group $G$ is given by

$$
\mathbb{F}_{j}(G)=F_{j}(\Gamma) \times L_{j}(\pi)
$$

as a set with multiplication obtained from restriction of the formula

$$
(f, x) \cdot(g, y)=\left(f \cdot g, \phi(g)^{-1}(x) \cdot y\right)
$$

for $f, g$ in $\Gamma$, and $x, y$ in $\pi$. 
Remark 2.10. To be precise, it must be checked that the stated multiplication in Definition 2.9 restricts to give $\mathbb{F}_{j}(G)$ as a subgroup of $G$. This verification is carried out in section 4 .

Let $H$ denote a discrete group. Recall that the commutator function

$$
[-,-]: H \times H \rightarrow H
$$

induces the structure of Lie algebra on the associated graded for the descending central series filtration of $H$. Kohno [10], and Falk-Randell [6] obtained a structure theorem for these Lie algebras restricted to certain semi-direct products of groups. A similar theorem holds for the mod- $p$ descending central series filtration [3]. However, there are other natural filtrations for which a similar extension theorem holds which are addressed by using the following definition.

Definition 2.11. A filtration of the group $H$ given by $\left\{F_{j}(H)\right\}$ is said to be Lie-like provided the commutator function

$$
[-,-]: H \times H \rightarrow H
$$

restricts to

for all $p, q \geq 0$.

$$
[-,-]: F_{p}(H) \times F_{q}(H) \rightarrow F_{p+q}(H)
$$

An analogue of this last property for split group extensions is defined next.

Definition 2.12. Consider the split extension

$$
1 \longrightarrow \pi \stackrel{i}{\longrightarrow} G \longrightarrow \Gamma \longrightarrow 1
$$

Two filtrations $L_{*}(\pi)$ and $F_{*}(\Gamma)$ are said to be stably Lie-like if

(1) $F_{*}(\Gamma)$ is Lie-like

(2) For $(f, x) \in F_{r}(\Gamma) \times L_{r}(\pi)$ and $(g, y) \in F_{s}(\Gamma) \times L_{s}(\pi), f(x \cdot g(y)) \in L_{r+s}(\pi)$

Remark 2.13. If $L_{*}(\pi)$ is a filtration as part of a stably Lie-like extension, then it is Lie-like. For this, notice that $(1, x) \in F_{r}(\Gamma) \times L_{r}(\pi)$ and $(1, y) \in F_{s}(\Gamma) \times L_{s}(\pi)$ implies that $x y \in L_{r+s}(\pi)$. Similary, $x^{-1} y^{-1} \in L_{r+s}(\pi)$. Thus, the commutator $[x, y]=x y x^{-1} y^{-1} \in L_{r+s}(\pi)$.

Theorem 2.14. Assume that the split extension

$$
1 \longrightarrow \pi \stackrel{i}{\longrightarrow} G \stackrel{p_{\phi}}{\longrightarrow} \Gamma \longrightarrow 1
$$

is classified by the map $\phi: \Gamma \rightarrow$ Aut $(\pi)$ which satisfies the following conditions: 
(1) The groups $\Gamma$ and $\pi$ admit filtrations (not necessarily bounded $p$-congruence systems) $F_{*}(\Gamma)$ and $L_{*}(\pi)$ as given in Definition 2.1.

(2) The filtrations for the groups $\pi$ and $\Gamma$ in part (1) are stable in the sense of Definition 2.5.

(3) The filtrations $F_{*}(\Gamma)$ and $L_{*}(\pi)$ are both stably Lie-like with associated graded Lie algebras denoted $g r_{*}^{F}(\Gamma)$ and $g r_{*}^{L}(\pi)$.

Then the filtration of G given in Definition 2.9 is Lie-like. Furthermore, there is a split, short exact sequence of Lie algebras

$$
0 \rightarrow g r_{*}^{L}(\pi) \rightarrow g r_{*}(G) \rightarrow g r_{*}^{F}(\Gamma) \rightarrow 0
$$

where $\operatorname{gr}_{*}(G)$ is the associated graded Lie algebra with Lie bracket induced by the commutator pairing

$$
[-,-]: G \times G \rightarrow G
$$

A systematic setting for stable extensions arises by considering automorphisms of a tower of groups given by a bounded $p$-congruence system for the group $\pi$. That method is recorded in the next section.

\section{Automorphisms of Towers of Groups}

The purpose of this section is (i) to define the automorphism group of a tower of groups over a discrete group $\pi$ and (ii) to show how the structure of the automorphism group of certain towers over $\pi$ gives rise to linear groups. The automorphism group of a tower of groups is defined next and is analogous to that of $[15]$.

Definition 3.1. A tower of groups over $\pi$ is

(1) a family of groups $L_{n}(\pi)$ for $n$ in a pointed, totally ordered index set $I=S \cup\{\bullet\}$ with unique least element $\bullet$ and $L_{\bullet}(\pi)=\pi$,

(2) for every $i \geq j \in I$, there is a (possibly empty ) family of homomorphisms $\mathcal{F}(i, j)$ given by $\alpha(i, j): L_{i}(\pi) \rightarrow L_{j}(\pi)$ with unique homomorphisms $\alpha(i, \bullet): L_{i}(\pi) \rightarrow \pi$ such that

$$
\alpha(i, \bullet)=\alpha(j, \bullet) \circ \alpha(i, j)
$$

for all $\alpha(i, j) \in \mathcal{F}(i, j)$. 
The automorphism group of this tower over $\pi$ denoted

$$
\operatorname{Aut}\left(L_{*}(\pi)\right)
$$

is the subgroup of elements $\left(\phi_{n}\right) \in \prod_{n \in I} A u t\left(L_{n}(\pi)\right)$ such that

$$
\phi_{j} \circ \alpha(i, j)=\alpha(i, j) \circ \phi_{i} \text {, for all } \alpha(i, j) \in \mathcal{F}(i, j) .
$$

A special case is given next.

Definition 3.2. An inductive tower of groups over $\pi$ is a tower of groups $\left\{L_{n}(\pi) \mid n \in\right.$ $I \cup\{\bullet\}\}$ over $\pi$ such that

(1) the index set $I$ is given by the natural numbers $\mathbb{N}=I$ with $\bullet=0$,

(2) each group $L_{n}(\pi)$ is a subgroup of $\pi$, and

(3) for every $i \geq j$, there is exactly one $\alpha(i, j): L_{i}(\pi) \rightarrow L_{j}(\pi)$ given by the natural inclusion.

Three remarks are given next.

Remark 3.3. $\quad$ (1) A filtration of $\pi$ given by

$$
\cdots \subseteq L_{j}(\pi) \subseteq \cdots \subseteq L_{1}(\pi) \subseteq L_{0}(\pi)=\pi
$$

is an inductive tower over $\pi$. Thus, a bounded $p$-congruence system is an inductive tower over $\pi$.

(2) The automorphism group of an inductive tower of groups over $\pi$ is the subgroup of elements in $A u t(\pi)$ which leave every $L_{n}(\pi)$ invariant.

(3) Restrict to the case where $L_{n}(\pi)$ is the $(n+1)$-st stage of the descending central series of $\pi, \Gamma^{n+1}(\pi)$. The natural inclusions

$$
\cdots \subseteq \Gamma^{n+1}(\pi) \subseteq \cdots \subseteq \Gamma^{2}(\pi) \subseteq \Gamma^{1}(\pi)=\pi
$$

specify an inductive tower over $\pi$ for which each $\Gamma^{n+1}(\pi)$ is invariant. Thus, the automorphism group of the inductive tower given by the descending central series is equal to $\operatorname{Aut}(\pi)$. Similar remarks apply to the mod- $p$ descending central series of $\pi$.

The next Lemma is a remark which follows from the above definitions.

Lemma 3.4. Assume that $\left\{L_{n}(\pi) \mid n \in I\right\}$ is an inductive tower of groups over $\pi$ so that the automorphism group of this tower, Aut $\left(L_{*}(\pi)\right)$, is a subgroup of $\operatorname{Aut}(\pi)$. Given an automorphism $\rho \in \operatorname{Aut}\left(L_{*}(\pi)\right)$, there is the natural induced split extension 
classified by regarding $\rho \in \operatorname{Aut}(\pi)$.

$$
1 \longrightarrow \pi \stackrel{i}{\longrightarrow} G \stackrel{p_{\rho}}{\longrightarrow} \Gamma \longrightarrow 1
$$

Automorphisms of certain towers then have implications for whether extensions are linear.

Theorem 3.5. Consider the split extension

$$
1 \longrightarrow \pi \longrightarrow G \longrightarrow \Gamma
$$

and suppose that the following conditions are satisfied:

(1) The filtration $L_{*}(\pi)$ is a Lubotzky filtration for the group $\pi$.

(2) The extension is classfied by a map $\rho: \Gamma \longrightarrow \operatorname{Aut}\left(L_{*}(\pi)\right)$ where $\operatorname{Aut}\left(L_{*}(\pi)\right)$ $\subseteq \operatorname{Aut}(\pi)$ is the automorphism group of the tower $L_{*}(\pi)$.

(3) There exists a Lubotzky filtration $F_{*}(\Gamma)$ for the group $\Gamma$ such that the filtrations $F_{*}(\Gamma)$ and $L_{*}(\pi)$ satisfy condition (2) in Definition 2.5.

Then $G$ is linear.

Proof. It suffices to show that the extension is stable in the sense of Definition 2.5 , since the result will then follow from Theorem 2.7

Suppose $(f, x) \in F_{r}(\Gamma) \times L_{r}(\pi)$ and $(g, y) \in F_{r+s}(\Gamma) \times L_{r+s}(\pi)$ where $r, s \geq 0$. Since the action of $\Gamma$ is tower-preserving and $y \in L_{r+s}(\pi)$, it follows that $f(y) \in$ $L_{r+s}(\pi)$ and the extension is stable.

Remark 3.6. The constructions in this section give a method to extend the techniques here to arbitrary group extensions without the assumption that the extension is required to be split. This remark will be addressed elsewhere.

\section{Two Filtrations}

The purpose of this section is to investigate split extensions equipped with two filtrations as given in Definition 2.5. Suppose

$$
1 \longrightarrow \pi \stackrel{i}{\longrightarrow} G \stackrel{p_{\phi}}{\longrightarrow} \Gamma \longrightarrow 1
$$

is a split extension classified by the map $\phi: \Gamma \rightarrow \operatorname{Aut}(\pi)$ together with filtrations 
(1) $L_{*}(\pi)$ given by

$$
\cdots \subseteq L_{j}(\pi) \subseteq \cdots \subseteq L_{1}(\pi) \subseteq L_{0}(\pi)=\pi
$$

for $j \geq 0$ for the group $\pi$ and

(2) $F_{*}(\Gamma)$ given by

$$
\cdots \subseteq F_{j}(\Gamma) \subseteq \cdots \subseteq F_{1}(\Gamma) \subseteq F_{0}(\Gamma)=\Gamma
$$

for $j \geq 0$ for the group $\Gamma$.

Assume that the extension (together with the two filtrations) is stable as in Definition 2.5. An equivalent technical formulation for the definition of a stable extension is stated next. Although elementary, direct, and technical, the next lemma is checked here as the second condition listed is the one actually used in the proofs of the theorems below.

Lemma 4.1. Assume that every $(g, y)$ in $F_{r+s}(\Gamma) \times L_{r+s}(\pi)$ and every $(f, x)$ in $F_{r}(\Gamma) \times L_{r}(\pi)$. The formulas given in Definition 2.5 by

(1) $f(y) \in L_{r+s}(\pi)$ and

(2) $g(x)=\delta_{x} \cdot x$ for $\delta_{x} \in L_{r+s}(\pi)$.

are equivalent to

(1) $f(y) \in L_{r+s}(\pi)$ and

(2) $g^{-1}(x) \cdot x^{-1} \in L_{r+s}(\pi)$.

Proof. Assume that every $(g, y)$ in $F_{r+s}(\Gamma) \times L_{r+s}(\pi)$ and every $(f, x)$ in $F_{r}(\Gamma) \times$ $L_{r}(\pi)$. It suffices to check that $g(x)=\delta_{x} \cdot x$ for $\delta_{x} \in L_{r+s}(\pi)$ if and only if $g^{-1}(x) \cdot x^{-1} \in L_{r+s}(\pi)$.

(1) Assume that $g^{-1}(x) \cdot x^{-1} \in L_{r+s}(\pi)$, and so $g^{-1}(x) \cdot x^{-1}=\epsilon_{x} \in L_{r+s}(\pi)$. Thus $g\left(\epsilon_{x}\right)=\delta_{x}{ }^{-1} \in L_{r+s}(\pi)$ by setting $f=g$ and $y=\epsilon_{x}$. Thus $x \cdot g\left(x^{-1}\right)=\delta_{x}^{-1}$.

(2) Assume that $g^{-1}(x) \cdot x^{-1}=\epsilon_{x}^{-1} \in L_{r+s}(\pi)$. Apply $g$ to obtain $x \cdot g\left(x^{-1}\right)=$ $g\left(g^{-1}(x) \cdot x^{-1}\right)=g\left(\epsilon^{-1}\right) \in L_{r+s}(\pi)$.

A filtration of $G, \mathbb{F}_{*}(G)$, was defined in Definition 2.9 without verifying that it is a filtration, namely $\mathbb{F}_{j}(G)$ is naturally a subgroup of $G$. This fact is recorded next. 
Lemma 4.2. Assume that

$$
1 \longrightarrow \pi \stackrel{i}{\longrightarrow} G \stackrel{p_{\phi}}{\longrightarrow} \Gamma \longrightarrow 1
$$

is a split extension classified by the map $\phi: \Gamma \rightarrow \operatorname{Aut}(\pi)$ and which is stable with respect to filtrations $L_{*}(\pi)$ and $F_{*}(\Gamma)$. Then $\mathbb{F}_{j}(G)$ is a group which is naturally a subgroup of $G$ and there is a morphism of extensions

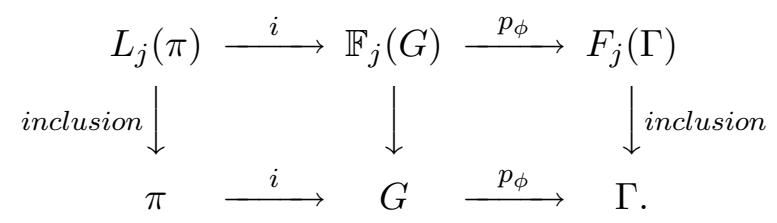

Proof. It suffices to check that $\mathbb{F}_{j}(G)$ is closed with respect to the product in $G$ given by $(f, x) \cdot(g, y)=\left(f \cdot g, \phi(g)^{-1}(x) \cdot y\right)$ for $f, g$ in $\Gamma$, and $x, y$ in $\pi$ where, by convention,

$$
g(x)=\phi(g)(x)
$$

Assume that $f, g$ are in $F_{j}(\Gamma)$, and that $x, y$ are in $L_{j}(\pi)$. By the "stability" condition in Definition 2.5, $\phi(g)^{-1}(x)$ is in $L_{j}(\pi)$. Thus $\phi(g)^{-1}(x) \cdot y$ is in $L_{j}(\pi)$. The lemma follows by inspection.

Properties of the groups $\mathbb{F}_{j}(G)$ are recorded in the next lemma.

Lemma 4.3. Let

$$
1 \longrightarrow \pi \stackrel{i}{\longrightarrow} G \stackrel{p_{\phi}}{\longrightarrow} \Gamma \longrightarrow 1
$$

denote a split extension classified by a map $\phi: \Gamma \rightarrow A u t(\pi)$ and which is stable with respect to filtrations $L_{*}(\pi)$ and $F_{*}(\Gamma)$. Let $\mathbb{F}_{j}(G)$ denote the groups defined earlier.

Then there are morphisms of split extensions

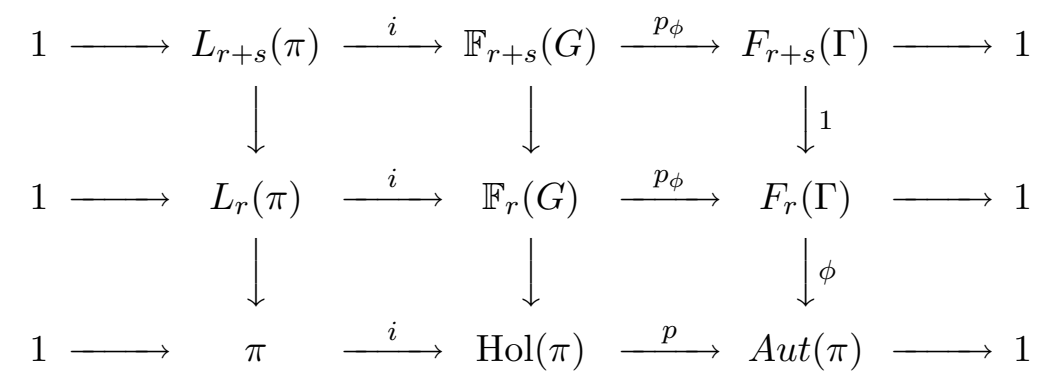


for every $s \geq 0$. Furthermore, $\mathbb{F}_{r+s}(G)$ is a normal subgroup of $\mathbb{F}_{r}(G)$ and there is an extension

$1 \longrightarrow L_{r}(\pi) / L_{r+s}(\pi) \stackrel{i}{\longrightarrow} \mathbb{F}_{r}(G) / \mathbb{F}_{r+s}(G) \stackrel{p_{\phi}}{\longrightarrow} F_{r}(\Gamma) / F_{r+s}(\Gamma) \longrightarrow 1$.

Thus if $F_{r}(\Gamma) / F_{r+s}(\Gamma)$ is generated by c elements and $L_{r}(\pi) / L_{r+s}(\pi)$ is generated by d elements, then $\mathbb{F}_{r}(G) / \mathbb{F}_{r+s}(G)$ is generated by $c+d$ elements.

Proof. In the proof below, recall the convention that $f(x)=\phi(f)(x)$ for $x \in \pi$, $f \in \Gamma$ and $\phi: \Gamma \rightarrow A u t(\pi)$. Since the split extension

$$
1 \longrightarrow \pi \stackrel{i}{\longrightarrow} G \stackrel{p_{\phi}}{\longrightarrow} \Gamma \longrightarrow 1
$$

is classified by a map $\phi: \Gamma \rightarrow A u t(\pi)$ which is stable with respect to filtrations $L_{*}(\pi)$ and $F_{*}(\Gamma)$, there is a morphism of split extensions

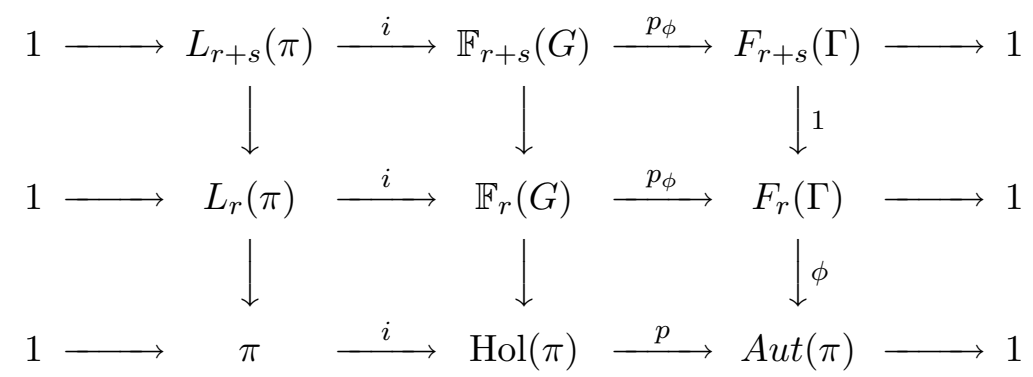

by Lemma 4.3 .

To check that $\mathbb{F}_{r+s}(G)$ is a normal subgroup of $\mathbb{F}_{r}(G)$ for any $s \geq 0$, let $(f, x)$ denote an element in $F_{r}(\Gamma) \times L_{r}(\pi)$ and $(g, y)$ an element in $F_{r+s}(\Gamma) \times L_{r+s}(\pi)$. Then

$$
(f, x) \cdot(g, y) \cdot(f, x)^{-1}=\left(f \cdot g \cdot f^{-1}, f\left(g^{-1}(x) \cdot y\right) \cdot f\left(x^{-1}\right)\right) .
$$

Notice that

(1) $f \cdot g \cdot f^{-1}$ is in $F_{r+s}(\Gamma)$ since it's a normal subgroup of $F_{r}(\Gamma)$,

(2) $f\left(g^{-1}(x) \cdot y\right) \cdot f\left(x^{-1}\right)=f\left(g^{-1}(x)\right) \cdot f(y) \cdot f\left(x^{-1}\right)$,

(3) $f\left(g^{-1}(x)\right) \cdot f\left(x^{-1}\right)$ is in $L_{r+s}(\pi)$ by stability,

(4) $y$ is in $L_{r+s}(\pi)$ by assumption, thus $f(y)$ is in $L_{r+s}(\pi)$ by stability,

(5) $f(x) \cdot f(y) \cdot f\left(x^{-1}\right)$ is in $L_{r+s}(\pi)$ by stability and

(6) $f\left(g^{-1}(x)\right) \cdot f(y) \cdot f\left(x^{-1}\right)=f\left(g^{-1}(x)\right) \cdot f\left(x^{-1}\right) \cdot f(x) \cdot f(y) \cdot f\left(x^{-1}\right)$ is in $L_{r+s}(\pi)$.

(7) Thus $\mathbb{F}_{r+s}(G)$ is a normal subgroup of $\mathbb{F}_{r}(G)$. 
Since $\mathbb{F}_{r+s}(G)$ is a normal subgroup of $\mathbb{F}_{r}(G)$, there is a morphism of extensions

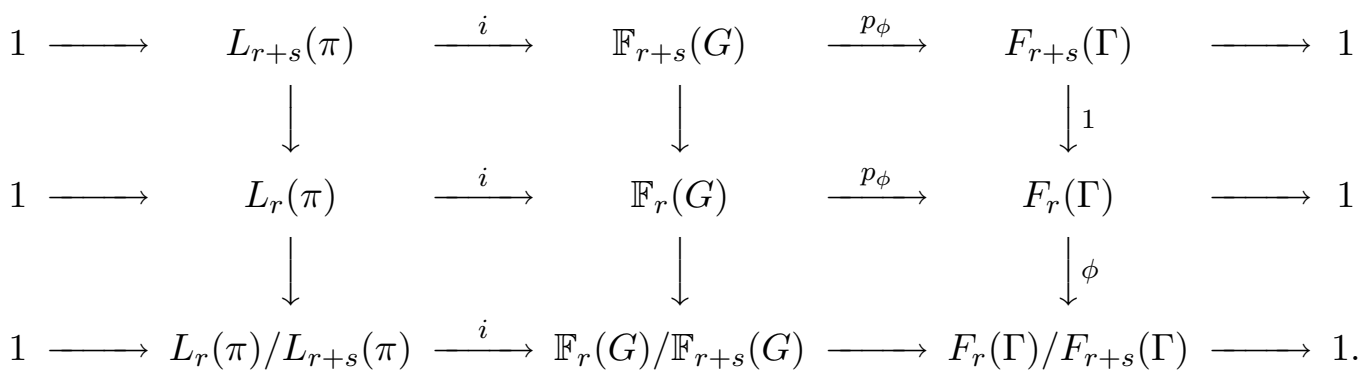

Since $F_{r}(\Gamma) / F_{r+s}(\Gamma)$ is generated by $d$ elements, the subgroup of $\mathbb{F}_{r}(G) / \mathbb{F}_{r+s}(G)$ generated by lifts of these elements together with $c$ elements which generate the kernel then generate the entire group. The lemma follows.

\section{Two Filtrations Continued: Proof of Theorem 2.7}

The purpose of this section is to describe properties of filtrations arising in section 4 inspired by work of A. Lubotzky who gave a sufficient condition for the existence of a finite dimensional faithful representation of a discrete group [13]. Lubotzky's filtration condition is changed below to fit questions for an extension theorem.

Given filtrations for $\Gamma$ and $\pi$ which are stable for the group extension

$$
1 \longrightarrow \pi \stackrel{i}{\longrightarrow} G \stackrel{p_{\phi}}{\longrightarrow} \Gamma \longrightarrow 1,
$$

there are naturally associated semi-direct products $\mathbb{F}_{j}(G)$ defined in section 4 .

Properties of the groups $\mathbb{F}_{j}(G)$ are recorded in the next lemma.

Lemma 5.1. Let

$$
1 \longrightarrow \pi \stackrel{i}{\longrightarrow} G \stackrel{p_{\phi}}{\longrightarrow} \Gamma \longrightarrow 1
$$

denote a split extension classified by a map $\phi: \Gamma \rightarrow A u t(\pi)$ and which is stable with respect to filtrations $L_{*}(\pi)$ and $F_{*}(\Gamma)$ which are also assumed to be $p$ congruence systems. Then $\mathbb{F}_{*}(G)$ is a p-congruence system for $G$.

Proof. To check that $\mathbb{F}_{*}(G)$ is $p$-congruence system for $G$, recall that it suffices to check (by Definition 2.2) that

$$
\bigcap_{j \geq 1} \mathbb{F}_{j}(G)=\{1\}
$$


and $\mathbb{F}_{*}(G)$ is a descending chain of normal subgroups

$$
\cdots \subseteq \mathbb{F}_{j}(G) \subseteq \cdots \subseteq \mathbb{F}_{1}(G) \subseteq \mathbb{F}_{0}(G)=G
$$

for $j \geq 0$ such that

(1) $G / \mathbb{F}_{1}(G)$ is finite and

(2) $\mathbb{F}_{1}(G) / \mathbb{F}_{1+j}(G)$ is a finite $p$-group for all $j \geq 0$.

That $\mathbb{F}_{r+s}(G)$ is a normal subgroup of $\mathbb{F}_{r}(G)$ is checked in Lemma 4.3. Notice that by the proof of Lemma 4.3,

$$
\bigcap_{j \geq 1} \mathbb{F}_{j}(G)=\bigcap_{j \geq 1}\left(F_{j}(\Gamma) \times L_{j}(\pi)\right)=\{1\} .
$$

Furthermore by $4.3, \mathbb{F}_{*}(G)$ is a decreasing filtration of $G$ with the property that there is an extension

$$
1 \longrightarrow L_{r}(\pi) / L_{r+s}(\pi) \stackrel{i}{\longrightarrow} \mathbb{F}_{r}(G) / \mathbb{F}_{r+s}(G) \stackrel{p_{\phi}}{\longrightarrow} F_{r}(\Gamma) / F_{r+s}(\Gamma) \longrightarrow 1 .
$$

Thus

(1) if $\Gamma / F_{j}(\Gamma)$ as well as $\pi / L_{j}(\pi)$ are finite, then so is $G / \mathbb{F}_{j}(G)$ and

(2) if $F_{r}(\Gamma) / F_{r+s}(\Gamma)$ as well as $L_{r}(\pi) / L_{r+s}(\pi)$ are finite $p$-groups, then so is $\mathbb{F}_{r}(G) / \mathbb{F}_{r+s}(G)$.

Thus $\mathbb{F}_{*}(G)$ is $p$-congruence system for $G$ and the lemma follows.

Lemma 5.2. Let

$$
1 \longrightarrow \pi \stackrel{i}{\longrightarrow} G \stackrel{p_{\phi}}{\longrightarrow} \Gamma \longrightarrow 1
$$

denote a split extension classified by a map $\phi: \Gamma \rightarrow$ Aut $(\pi)$ and which is stable with respect to filtrations $L_{*}(\pi)$ and $F_{*}(\Gamma)$ which are also assumed to be bounded $p$ congruence systems. Then $\mathbb{F}_{*}(G)$ is a Lubotzky filtration, a bounded p-congruence system.

Proof. By Lemma 5.1, $\mathbb{F}_{*}(G)$ is $p$-congruence system for $G$. Furthermore by 4.3, $\mathbb{F}_{*}(G)$ is a decreasing filtration of $G$ with the property that there is an extension

$1 \longrightarrow L_{r}(\pi) / L_{r+s}(\pi) \stackrel{i}{\longrightarrow} \mathbb{F}_{r}(G) / \mathbb{F}_{r+s}(G) \stackrel{p_{\phi}}{\longrightarrow} F_{r}(\Gamma) / F_{r+s}(\Gamma) \longrightarrow 1$. 
Thus if $F_{r}(\Gamma) / F_{r+s}(\Gamma)$ is generated by $c$ elements and $L_{r}(\pi) / L_{r+s}(\pi)$ is generated by $d$ elements, then $\mathbb{F}_{r}(G) / \mathbb{F}_{r+s}(G)$ is generated by $c+d$ elements. By Definition $2.3, \mathbb{F}_{*}(G)$ is a a Lubotzky filtration, a bounded $p$-congruence system for $G$.

One consequence of Theorem 2.4 as well as Lemma 5.1 is Theorem 2.7.

\section{Proof of Theorem 2.14}

Consider the two filtrations $L_{*}(\pi)$ and $F_{*}(\Gamma)$ associated to the stable extension

$$
1 \longrightarrow \pi \stackrel{i}{\longrightarrow} G \stackrel{p_{\phi}}{\longrightarrow} \Gamma \longrightarrow 1
$$

as stated in Definition 2.5 and developed in section 4 .

A filtration of $G$ regarded as a set was defined by

$$
\mathbb{F}_{j}(G)=F_{j}(\Gamma) \times L_{j}(\pi)
$$

in Definition 2.9. Some properties of $\mathbb{F}_{j}(G)$ were proven in Lemmas 4.2 and 4.3 as follows.

(1) The subset $\mathbb{F}_{j}(G)$ is naturally a subgroup of $G$.

(2) There is a morphism of split group extensions

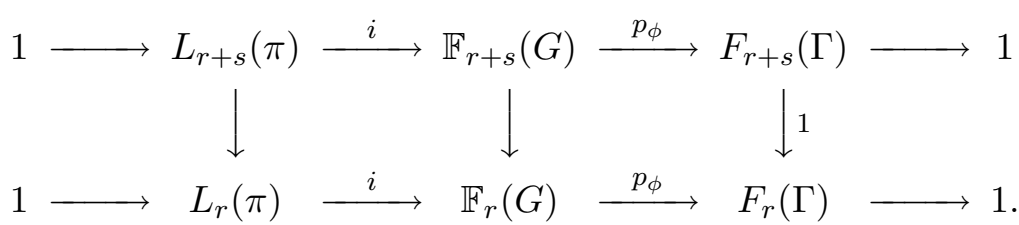

(3) There is a split extension

$1 \longrightarrow L_{r}(\pi) / L_{r+s}(\pi) \stackrel{i}{\longrightarrow} \mathbb{F}_{r}(G) / \mathbb{F}_{r+s}(G) \stackrel{p_{\phi}}{\longrightarrow} F_{r}(\Gamma) / F_{r+s}(\Gamma) \longrightarrow 1$.

Consider the filtration quotients

$g r_{r}^{F}(\Gamma)=F_{r}(\Gamma) / F_{r+1}(\Gamma), g r_{r}^{L}(\pi)=L_{r}(\pi) / L_{r+1}(\pi)$, and $\quad g r_{r}(G)=\mathbb{F}_{r}(G) / \mathbb{F}_{r+1}(G)$.

Then there is a split short exact sequence of groups

$$
\{0\} \longrightarrow g r_{r}^{L}(\pi) \longrightarrow g r_{r}(G) \longrightarrow g r_{r}^{F}(\Gamma) \longrightarrow\{0\}
$$


by Lemmas 4.2 and 4.3 .

That $\mathbb{F}_{*}(G)$ is Lie-like is checked next. Suppose $(f, x) \in \mathbb{F}_{s}(G)$ and $(g, y) \in$ $\mathbb{F}_{r}(G)$. It will be checked that $g\left[f\left(g^{-1}(x) \cdot y\right) \cdot f\left(x^{-1}\right)\right] \cdot g\left(y^{-1}\right) \in L_{r+s}(\pi)$ whenever the following conditions are satisfied:

(1) The extenstion is stable.

(2) The filtration on $\Gamma$ is Lie-like.

(3) $f(x \cdot g(y)) \in L_{r+s}(\pi)$.

Since the filtration $F_{*}(\Gamma)$ is Lie-like, there exists $h \in F_{r+s}(\Gamma)$ with $g f g^{-1}=f h$. Since the extension is stable, there exists $\delta_{x} \in L_{r+s}(\pi)$ such that $h(x)=\delta_{x} \cdot x$. This implies the following:

$$
\begin{aligned}
g\left[f\left(g^{-1}(x) \cdot y\right) \cdot f\left(x^{-1}\right)\right] \cdot g\left(y^{-1}\right) & =g f g^{-1}(x) \cdot g f(y) \cdot g f\left(x^{-1}\right) \cdot g\left(y^{-1}\right) \\
& =f h(x) \cdot g f(y) \cdot g f\left(x^{-1}\right) \cdot g\left(y^{-1}\right) \\
& =f\left(\delta_{x}\right) \cdot f(x) \cdot g f(y) \cdot g f\left(x^{-1}\right) \cdot g\left(y^{-1}\right)
\end{aligned}
$$

Notice that $f\left(\delta_{x}\right) \in L_{r+s}(\pi)$ by stability. So it suffices to show $f(x) \cdot g f(y)$. $g f\left(x^{-1}\right) \cdot g\left(y^{-1}\right) \in L_{r+s}(\pi)$. Since $F_{*}(\Gamma)$ is Lie-like, there exists $k \in F_{r+s}(\Gamma)$ with $g f=f g k$. Since the extension is stable and the filtration of $\pi$ is given by normal subgroups, there is $\delta_{y} \in L_{r+s}(\pi)$ such that $k(y)=y \cdot \delta_{y}$. This implies the following:

$$
\begin{aligned}
f(x) \cdot g f(y) \cdot g f\left(x^{-1}\right) \cdot g\left(y^{-1}\right) & =f(x) \cdot f g k(y) \cdot g f\left(x^{-1}\right) \cdot g\left(y^{-1}\right) \\
& =f(x) \cdot f g\left(y \cdot \delta_{y}\right) \cdot g\left(f\left(x^{-1}\right) \cdot y^{-1}\right) \\
& =f(x) \cdot f g(y) \cdot f g\left(\delta_{y}\right) \cdot g\left(f\left(x^{-1}\right) \cdot y^{-1}\right) \\
& =f(x \cdot g(y)) \cdot f g\left(\delta_{y}\right) \cdot g\left(f\left(x^{-1}\right) \cdot y^{-1}\right)
\end{aligned}
$$

Now $f g\left(\delta_{y}\right) \in L_{r+s}(\pi)$ by the stability condition. The additional condition (3) above gives that $f(x \cdot g(y)) \in L_{r+s}(\pi)$ and $g\left(f\left(x^{-1}\right) \cdot y^{-1}\right) \in L_{r+s}(\pi)$. 
To finish the proof, notice that Theorem 2.14 follows at once from the property that these maps induce morphisms of Lie algebras, a property which is checked next.

First observe that if $x \in L_{r}(\pi)$ and $y \in L_{s}(\pi)$, then $[x, y] \in L_{r+s}(\pi)$ by the assumption that the filtration $L_{*}(\pi)$ is Lie-like. Secondly, since the filtration of $G$ is Lie-like, there is a commutative diagram

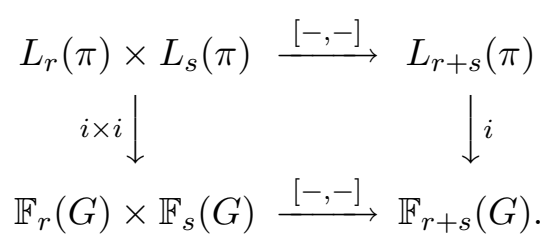

Thus the map $i: \pi \rightarrow G$ passes to quotients on the level of associated graded modules and preserves the structure of the underlying Lie algebras. Thus the map

$$
p_{\phi}: G \rightarrow \Gamma
$$

preserves the structure of Lie algebras. The Theorem follows.

\section{An EXAmple}

The purpose of this section is to give examples of Theorem 2.7 and Theorem 3.5. This example has the serious drawback that the extension can be shown to be linear by a "bare-hands", more general, classical argument which is reviewed in section 8 .

These examples arise by forming the split extension

$$
1 \rightarrow F_{n} \rightarrow G \rightarrow \Gamma \rightarrow 1
$$

where

(1) $\Gamma$ is a subgroup of $G L(2, \mathbb{Z})$ ( and thus $\Gamma$ has a normal finite index subgroup which is free ),

(2) $F_{n}$ is isomorphic to a principal congruence subgroup of level $p^{r}$ in $P S L(2, \mathbb{Z})$, and

(3) $\Gamma$ acts by conjugation on $F_{n}$. 
Let $P \Gamma\left(2, p^{r}\right)$ denote the kernel of the "mod- $p^{r}$ reduction map"

$$
\rho_{p^{r}}: P S L(2, \mathbb{Z}) \longrightarrow P S L\left(2, \mathbb{Z} / p^{r} \mathbb{Z}\right) .
$$

Natural automorphisms of $P \Gamma\left(2, p^{r}\right)$ as well as the tower

$$
\cdots \subseteq P \Gamma\left(2, p^{r+1}\right) \subseteq P \Gamma\left(2, p^{r}\right) \subseteq \cdots \subseteq P \Gamma(2, p)=\pi
$$

are given by conjugation by an element in $G L(2, \mathbb{Z})$.

Furthermore if $p$ is a prime, the groups $P \Gamma(2, p)$ are free on $1+p\left(p^{2}-1\right) / 12$ generators if $p$ is an odd prime [8] or 2 letters if $p=2$ [9]. Let $\Gamma\left(2, p^{r}\right)$ denote the kernel of the natural reduction map $G L(2, \mathbb{Z}) \rightarrow G L\left(2, \mathbb{Z} / p^{r} \mathbb{Z}\right)$. Below it is shown that

$$
\cdots P \Gamma\left(2, p^{r+1}\right) \subseteq P \Gamma\left(2, p^{r}\right) \subseteq \cdots \subseteq P \Gamma\left(2, p^{2}\right) \subseteq P \Gamma(2, p)
$$

gives a Lubotzky filtration for $P \Gamma(2, p)$. The reader can check that similar arguments show that

$$
\cdots \subseteq \Gamma\left(2, p^{r+1}\right) \subseteq \Gamma\left(2, p^{r}\right) \subseteq \cdots \subseteq \Gamma\left(2, p^{2}\right) \subseteq \Gamma(2, p)
$$

gives a Lubotzky filtration for $\Gamma(2, p)$. This information is recorded next while a more standard development is given in section 8 .

Lemma 7.1. The filtration

$$
\cdots \subseteq \Gamma\left(2, p^{r+1}\right) \subseteq \Gamma\left(2, p^{r}\right) \subseteq \cdots \subseteq \Gamma\left(2, p^{2}\right) \subseteq \Gamma(2, p)
$$

of $\Gamma(2, p)$ is a Lubotzky filtration.

Proposition 7.2. The extension

$$
1 \longrightarrow P \Gamma\left(2, p^{r}\right) \longrightarrow G \longrightarrow \Gamma\left(2, p^{s}\right) \longrightarrow 1
$$

is linear where $\Gamma\left(2, p^{s}\right)$ acts on $P \Gamma\left(2, p^{r}\right)$ by conjugation and $r, s \geq 1$. 
Proof. Let $f \in \Gamma\left(2, p^{s}\right)$ and $x \in P \Gamma\left(2, p^{r+q}\right)$ where $q \geq 0$, so that $x$ projects to the identity in $P S L\left(2, \mathbb{Z} / p^{r+q} \mathbb{Z}\right)$. Since $f x f^{-1} \in P \Gamma\left(2, p^{r+q}\right)$, the conjugation action is tower-preserving. Thus this filtration, along with the filtration of $P \Gamma\left(2, p^{r}\right)$ is stable in the sense of Definition 2.5. Theorem 2.7 then implies that $G$ is linear.

Additional properties, some classical, some possibly not, are recorded next. Notice that an automorphism of the tower of groups

$$
\cdots \subseteq P \Gamma\left(2, p^{r+1}\right) \subseteq P \Gamma\left(2, p^{r}\right) \subseteq \cdots \subseteq P \Gamma(2, p)=\pi
$$

induces an automorphism of the Lie algebra

$$
g r_{*}(P \Gamma(2, p))=\oplus_{s \geq 1} P \Gamma\left(2, p^{s}\right) / P \Gamma\left(2, p^{s+1}\right) .
$$

Thus it is natural to identify the structure of this Lie algebra.

That structure is given next where related, and standard properties of these principal congruence subgroups are recorded for convenience. Recall that $P \Gamma\left(2, p^{s+1}\right)$ is a normal subgroup of $P \Gamma\left(2, p^{s}\right)$. Define

$$
g r_{s}(P \Gamma(2, p))=P \Gamma\left(2, p^{s}\right) / P \Gamma\left(2, p^{s+1}\right)
$$

the associated graded.

The commutator map

$$
[-,-]: P S L(2, \mathbb{Z}) \times P S L(2, \mathbb{Z}) \rightarrow P S L(2, \mathbb{Z})
$$

restricts to

$$
[-,-]: P \Gamma\left(2, p^{r}\right) \times P \Gamma\left(2, p^{s}\right) \rightarrow P \Gamma\left(2, p^{r+s}\right),
$$

and induces the structure of Lie algebra on the associated graded

$$
\left.g r_{*}(P \Gamma(2, p))\right)=\oplus_{s \geq 1} g r_{s}(P \Gamma(2, p))
$$

with

$$
[-,-]: g r_{s}(P \Gamma(2, p)) \otimes g r_{t}(P \Gamma(2, p)) \rightarrow g r_{s+t}(P \Gamma(2, p))
$$


Furthermore, the $p$-th power map

$$
\psi^{p}: P \Gamma\left(2, p^{s}\right) \rightarrow P \Gamma\left(2, p^{s+1}\right)
$$

induces a ( possibly non-linear ) map

$$
\psi^{p}: g r_{s}(P \Gamma(2, p)) \rightarrow g r_{s+1}(P \Gamma(2, p)) .
$$

Together with the previous structure of Lie algebra for $g r_{*}(P \Gamma(2, p))$, this gives the structure of a restricted Lie algebra over the field with $p$ elements $\mathbb{F}_{p}$. Classical, well-known properties of the filtration quotients $P \Gamma\left(2, p^{r}\right) / P \Gamma\left(2, p^{r+1}\right)$ are recorded in the next theorem.

Theorem 7.3. If $p$ is an odd prime, there are isomorphisms

$$
\theta_{q}: \oplus_{3} \mathbb{Z} / p \mathbb{Z} \rightarrow g r_{q}(P \Gamma(2, p))
$$

with a choice of basis given by

$$
A_{q}=\left(\begin{array}{cc}
1 & p^{q} \\
0 & 1
\end{array}\right), \quad B_{q}=\left(\begin{array}{cc}
1 & 0 \\
p^{q} & 1
\end{array}\right), \quad C_{q}=\left(\begin{array}{cc}
1+p^{q} & p^{q} \\
-p^{q} & 1-p^{q}
\end{array}\right) .
$$

Furthermore,

$$
B_{q} \cdot C_{q} \cdot A_{q}^{-1}=D_{q}
$$

( where the next matrix is not the reduction of a matrix in $P S L(2, \mathbb{Z})$ but represents a nontrivial coset in $\mathrm{gr}_{q}(P \Gamma(2, p))$ )

$$
D_{q}=\left(\begin{array}{cc}
1+p^{q} & 0 \\
0 & 1-p^{q}
\end{array}\right)
$$

If $p=2$, there are isomorphisms

$$
\theta_{q}: \oplus_{2} \mathbb{Z} / 2 \mathbb{Z} \rightarrow g r_{q}(P \Gamma(2,2))
$$

with a choice of basis given by

Furthermore

$$
A_{q}=\left(\begin{array}{ll}
1 & 2^{q} \\
0 & 1
\end{array}\right), \quad B_{q}=\left(\begin{array}{cc}
1 & 0 \\
2^{q} & 1
\end{array}\right)
$$

$$
\left[A_{q}, B_{q}\right]=1
$$


The additive structure given above is given in a global way in terms of restricted Lie algebras. That structure is listed next.

Theorem 7.4. If $p=2$, then the restricted Lie algebra $\operatorname{gr}_{*}(P \Gamma(2,2))$ is generated by $A_{1}$ and $B_{1}$ (as a restricted Lie algebra). Furthermore, $g r_{*}(P \Gamma(2,2))$ is the abelian, free restricted Lie algebra (over $\mathbb{F}_{2}$ ) generated by $A_{1}$ and $B_{1}$ where, redundantly, the following relations are satisfied:

(1) $\left[A_{q}, B_{s}\right]=1$ for all $q$ and $s$,

(2) $\psi^{2}\left(A_{q}\right)=A_{q+1}$ and

(3) $\psi^{2}\left(B_{q}\right)=B_{q+1}$.

If $p$ is an odd prime, then the restricted Lie algebra $\operatorname{gr}_{*}(P \Gamma(2, p))$ is generated by $A_{1}, B_{1}$ and $D_{1}$. Furthermore, $g r_{*}(P \Gamma(2, p))$ is the free restricted Lie algebra (over $\mathbb{F}_{p}$ ) generated by $A_{1}, B_{1}$ and $D_{1}$ subject to the following relations.

(1) $\left[A_{q}, B_{s}\right]=D_{q+s}$ for all $q$ and $s$,

(2) $\left[A_{q}, D_{s}\right]=A_{q+s}^{-2}$ for all $q$ and $s$,

(3) $\left[B_{q}, D_{s}\right]=B_{q+s}^{2}$ for all $q$ and $s$,

(4) $\psi^{p}\left(A_{q}\right)=A_{q+1}$,

(5) $\psi^{p}\left(B_{q}\right)=B_{q+1}$, and

(6) $\psi^{p}\left(D_{q}\right)=D_{q+1}$.

Theorem 7.3 is classical and can be found in [8]. The proof of Theorem 7.4 is a computation based on the next classical lemma.

Proposition 7.5. The quotient $P \Gamma\left(2, p^{r}\right) / P \Gamma\left(2, p^{r+1}\right)$ is isomorphic to the kernel of the natural reduction map

$$
\gamma_{p^{r}}: P S L\left(2, \mathbb{Z} / p^{r+1} \mathbb{Z}\right) \longrightarrow P S L\left(2, \mathbb{Z} / p^{r} \mathbb{Z}\right)
$$

and so there are isomorphisms

$$
P \Gamma\left(2, p^{r}\right) / P \Gamma\left(2, p^{r+1}\right) \cong \begin{cases}\oplus_{2} \mathbb{Z} / 2 \mathbb{Z} & \text { if } p=2, \text { and } \\ \oplus_{3} \mathbb{Z} / p \mathbb{Z} & \text { if } p \text { is an odd prime. }\end{cases}
$$

Proof. The proof follows directly from the commutative diagram where the rows and columns are all group extensions: 


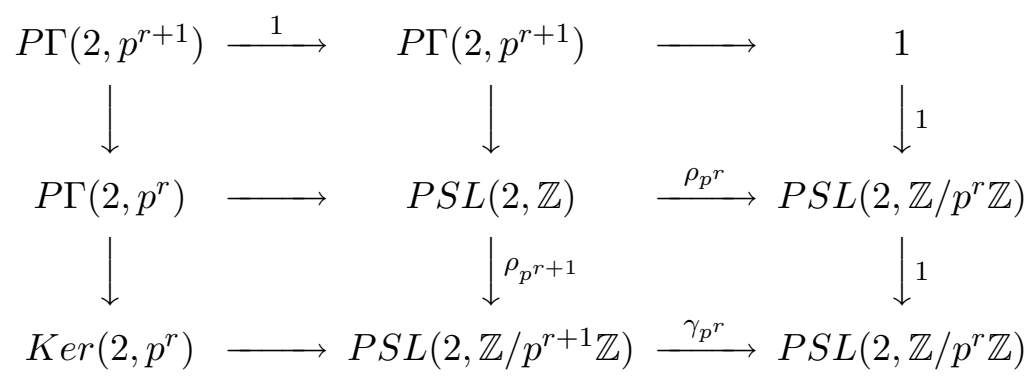

More applications to other groups $S L(n, A)$ and to their cohomology will appear in the thesis of J. Lopez [12].

\section{A Second Example}

The purpose of this section is to review classical properties of the natural extension of $P S L(n, A)$ by $S L(n, A)$ with conjugation action where $A$ is a commutative ring. First, Let $Z(G)$ denote the center of the group $G$ and consider the conjugation action of $G$ on itself thus inducing an action of $G / Z(G)$ on $G$ given by

$$
\operatorname{Inn}(G)=G / Z(G) \rightarrow \operatorname{Aut}(G) .
$$

Let $\Delta(G)$ denote the associated extension

$$
1 \longrightarrow G \stackrel{i}{\longrightarrow} \Delta(G) \stackrel{p}{\longrightarrow} G / Z(G) \longrightarrow 1
$$

obtained from the conjugation action of $G / Z(G)$ on $G$.

Notice that $S L(n, A)$ acts on the full matrix ring $M(n, A)$ in two ways recorded next where $M \in M(n, A)$ and $\gamma, y \in S L(n, A)$.

(1) $(1, y)(M)=y M$ and

(2) $(\gamma, 1)(M)=\gamma \cdot(M) \cdot \gamma^{-1}$.

Then define

$$
(\gamma, y)(M)=\gamma \cdot(y \cdot M) \cdot \gamma^{-1}
$$

Lemma 8.1. The formula

$$
(\gamma, y)(M)=\gamma \cdot(y \cdot M) \cdot \gamma^{-1}
$$


for $M \in M(n, A)$ and $\gamma, y \in S L(n, A)$ specifies a left action of $\Delta(S L(n, A))$ on $M(n, A)$.

Assume Lemma 8.1 for the moment.

Theorem 8.2. The formula

$$
\rho((\gamma, y))(M)=\gamma \cdot(y \cdot M) \cdot \gamma^{-1}
$$

for $M \in M(n, A)$ and $\gamma, y \in S L(n, A)$ induces a faithful representation

$$
\rho: \Delta(S L(n, A)) \rightarrow G L\left(n^{2}, A\right) .
$$

The theorem has an elementary, immediate consequence.

Corollary 8.3. If $G$ is a group with trivial center and is a subgroup of $S L(n, A)$, then the split extension

$$
1 \longrightarrow G \stackrel{i}{\longrightarrow} \Delta(G) \stackrel{p}{\longrightarrow} G / Z(G)=G \longrightarrow 1
$$

where $G$ acts on itself by conjugation is a subgroup of $G L\left(n^{2}, A\right)$.

The proof of Theorem 8.2 is given next.

Proof. First notice that by Lemma 8.1 the function $\rho$ is a homomorphism.

If $(\gamma, y)$ is in the kernel of $\rho$ then

$$
\rho((\gamma, y))(M)=M
$$

for all $M \in M(n, A)$. Let $M=1_{n}$ the multiplicative identity element in $M(n, A)$. Then

$$
\gamma \cdot\left(y \cdot 1_{n}\right) \cdot \gamma^{-1}=1_{n}
$$

implies that $y=1_{n}$.

Thus assume that $\left(\gamma, 1_{n}\right)$ is in the kernel of $\rho$. Hence

$$
\gamma(M) \gamma^{-1}=M
$$


for all $M \in M(n, A)$, and $\gamma$ is in the center of $P S L(n, A)$ which, by definition is trivial. The Theorem follows.

The proof of Lemma 8.1 is given next.

Proof. Let $(\alpha, x)$ and $(\beta, y)$ denote elements in the semi-direct product $\Delta(S L(n, A))$.

Then the following hold for $M \in M(n, A)$.

(1) $(\alpha, x)(\beta, y)=\left(\alpha \beta, \beta^{-1} x \beta y\right)$

(2) $\left(\alpha \beta, \beta^{-1} x \beta y\right)(M)=\alpha \beta\left(\beta^{-1} x \beta y M\right) \beta^{-1} \alpha^{-1}=\alpha(x \beta y M) \beta^{-1} \alpha^{-1}$

(3) $(\alpha, x)((\beta, y)(M))=(\alpha, x)\left(\beta y M \beta^{-1}\right)=\alpha\left(x \beta(y M) \beta^{-1}\right) \alpha^{-1}$

Since the two formulas agree, the Lemma follows.

\section{REFERENCES}

[1] S. Bigelow, Braid groups are linear, J. Amer. Math. Soc., 14 (2001), 471-486.

[2] D. Cohen, F. R. Cohen, S. Prassidis, Centralizers of Lie Algebras Associated to the Descending Central Series of Certain Poly-Free Groups, submitted.

[3] F. R. Cohen, and J. Wu, On braid groups, free groups, and the loop space of the 2-sphere, Progress in Mathematics, 215(2003), 93-105, Birkhaüser, and Braid groups, free groups, and the loop space of the 2-sphere, math.AT/0409307.

[4] H. S. M. Coxeter, W. O. J. Moser, Generators and relations for discrete groups, SpringerVerlag, Berlin, 1957.

[5] J. D. Dixon, M. P. F. Du Sautoy, A. Mann and D. Segal, Analytic Pro-P Groups, 2nd Edition Series: Cambridge Studies in Advanced Mathematics (No. 61), (ISBN-13: 9780521542180 - ISBN-10: 0521542189).

[6] M. Falk, and R. Randell, The lower central series of a fiber-type arrangement, Invent. Math., 82 (1985), 77-88.

[7] E. Formanek, C. Procesi, The automorphism group of a free group is not linear, J. Algebra, 149 (1992), 494-499.

[8] H. Frasch, Die Erzeugenden der Hauptkongruenzgruppen für Primastahlstufen, Math. Annalen, 20(1882),229-251.

[9] F. Klein, Gesammelte mathematische Abhandlungen, Springer-Verlag, Berlin, 1923.

[10] T. Kohno, Série de Poincaré-Koszul associée aux groupes de tresses pures, Inv. Math., 179 (1985).

[11] D. Krammer, Braid groups are linear, Ann. of Math. 155 (2002), 131-156.

[12] J. Lopez, On Lie Algebras and Cohomology Associated to Congruence Subgroups, Ph.D. Thesis, University of Rochester, April 2010. 
[13] A. Lubotzky, A group theoretic characterization of linear groups, J. Algebra, 113 (1988), 207-214.

[14] J. McCool, On basis-conjugating automorphisms of free groups, Canadian J. Math., vol. 38,12(1986), 1525-1529.

[15] L. Schneps, Groupe de Grothendieck-Teichmüller et automorphsimes de groupes de tresses, website.

[16] M. Voloshina, Thesis, U. Rochester, 2003.

F. R. Cohen

Department of Mathematics

University of Rochester

Rochester, NY 14627

E-mail: cohf@math.rochester.edu

\section{Marston Conder}

Department of Mathematics

New Zealand Institute of Mathematics and its Applications

E-mail: m.conder@auckland.ac.nz

\section{J. Lopez}

Department of Mathematics

University of Rochester

Rochester, NY 14627

E-mail: jlopez@math.rochester.edu

Stratos Prassidis

Department of Mathematics

Canisius College

Buffalo, NY 14208, U.S.A.

E-mail: prasside@canisius.edu 